against the commensal microflora in the gut. Consistent with this hypothesis, IL-23 is required for gut inflammation via innate immune mechanisms in T cell-deficient animals ${ }^{9}$. This finding clearly shows that IL-23 may cause gut inflammation in the absence of T lymphocytes, further highlighting a master control function of IL-23 in the gut.

Other recent research has suggested that ๑ both of these mechanisms may be operating. For instance, two recent studies in the Journal of Experimental Medicine begin to dissect the role of IL-23 in mastering the gut immune system. These studies suggest that IL-23 signaling in the gut directly controls pro-inflammatory cytokine production by cells of the mucosal immune system.

Hue et al. ${ }^{2}$ showed that antibodies to p19, a subunit thought to be specific to IL-23, suppress chronic intestinal inflammation in a model of IBD (T cell-deficient, Helicobacter hepaticus-infected RAG knockout mice). Knocking out p19 function seemed to inhibit the production of various pro-inflammatory cytokines such as tumor necrosis factor (TNF), IFN- $\gamma$, IL-1, IL-6 and IL- 17 .

These findings are consistent with those of Kullberg et al. ${ }^{3}$ who examined a mouse model of colitis, in which a subset of naive $\mathrm{T}$ lymphocytes is adoptively transferred into immunodeficient, RAG knockout hosts and causes chronic intestinal inflammation. The authors found that colitis was suppressed in recipient mice deficient in p19 and p40-but not in $\mathrm{p} 35$, the subunit thought to be specific for IL-12. Suppression of colitis was associated with reduced levels of pro-inflammatory mediators (TNF, IL- 6 and IFN- $\gamma$ ). In addition, p19 was found to regulate mucosal rather than systemic immune responses in this colitis model, suggesting a 'mucosa-selective' role of IL- $23^{2,3}$.

Both of these mouse studies dovetail with the work of Yen et al..$^{10}$ earlier this year. These authors found that colitis in another IBD animal model (IL-10-deficient mice) was dependent on IL-23 rather than IL-12, and could be exacerbated by the administration of recombinant IL-23.

Taken together, all these data show that IL-23 initiates and perpetuates both innate and $\mathrm{T}$ cell-mediated intestinal inflammation.

What are the implications of the new findings for the clinic? Active Crohn disease is associated with increased IL-12 and IL-23 production, and IL-12 may still have value as a drug target. Indeed, a controlled trial of patients with Crohn disease showed that an antibody to the p40 subunit of IL-12/IL-23 results in higher rates of clinical responses and

\title{
SCANNING THE GENOME
}

In the hands of geneticists, the old saw "each of us is unique" becomes a challenge. The human genetic landscape consists of 6 to 8 million common genetic variants, defined as those carried by at least $5 \%$ of the population-a subset of which contribute appreciably to the heritability of many common diseases. Only recently have researchers begun to rise to this challenge, typified in the study of Duerr et al. ${ }^{1}$

These authors are among the first to deploy a genome-wide association strategy to identify risk factors for common disease-in this case, Crohn disease [p 26]. Such genome-wide approaches have been used previously to identify genetic variants conferring risk for age-related macular degeneration ${ }^{2}$, and promise to accelerate the search for disease-associated polymorphisms in the future.

Whole-genome approaches have only recently become feasible through advances in high-throughput genotyping, which enable hundreds of thousands of markers to be genotyped in hundreds of individuals at an affordable cost, and the development of a reference panel of common genetic variation known as the HapMap ${ }^{3}$. With such tools, geneticists can examine a panel of genetic markers that effectively capture a substantial fraction of common variants indirectly, taking advantage of the fact that many variants are in 'linkage disequilibrium', or statistically correlated, in the human population ${ }^{3}$. The marker panel used by Duerr et al., for instance, consisted of 308,332 single nucleotide polymorphisms and is estimated to provide information on roughly $75 \%$ of the common variants present in populations of European ancestry ${ }^{4,5}$.

Key aspects of any genome-wide association study are the study design and analysis strategy. As with any epidemiological study, sample sizes must be sufficiently large to allow identification of risk factors, which, in the setting of common diseases, are generally expected to confer only small effects on overall risk. Balanced against this is the considerable cost of genotyping, at high density, large numbers of individuals. The study by Duerr et al. strikes a good balance, comparing more than 300,000 genetic markers in roughly 500 individuals with Crohn disease and 500 healthy controls.

The next key challenge, after controlling for potential sources of bias such as genotyping error, is to set an appropriate statistical threshold for deciding which of the many observed differences in marker frequency between affected individuals and controls are significant, a critical issue given the very large number of markers being examined. Duerr et al. applied a stringent statistical cutoff to minimize the risk of false positives, leaving them with three markers considered significant at a genome-wide level. As a final critical step in establishing the robustness of their results, Duerr et al. replicated the association between $I L 23 R$ and Crohn disease in two independent collections of samples.

In contrast to 'candidate gene' approaches, genome-wide association studies have a key advantage: they offer a fairly unbiased survey of the genome and make no a priori assumptions about where risk variants might reside. An important weakness is that current genotyping platforms are limited in their coverage of rare genetic variants, which may also contribute substantially to the genetic architecture of common diseases. At present, the latter are best examined through targeted resequencing efforts as exemplified by recent studies showing that rare varients in two genes, ATM and BRIP1, are associated with increased risk of familial breast cancer6,7. If the so-called ' $\$ 1,000$ genome' comes to fruition, these targeted efforts may soon give way to whole-genome resequencing that would reveal the complete spectrum of genetic variants underlying common diseases.

Kyle Vogan

1. Duerr, R.H. et al. Science 314, 1461-1463 (2006).

Kyle Vogan is a Senior Editor at Nature Genetics.

2. Klein, R.J. et al. Science 308, 385-389 (2005).

3. International HapMap Consortium. Nature 437, 1299-1320 (2005)

4. Barrett, J.C. \& Cardon, L.R. Nat. Genet. 38, 659-662 (2005).

5. Pe'er, I. et al. Nat. Genet. 38, 663-667 (2005).

6. Renwick, A. et al. Nat. Genet. 38, 873-875 (2006).

7. Seal, S. et al. Nat. Genet. 38, 1239-1241 (2006). 